\title{
Platelet Rich Plasma combined with an Electrospun Collagen Scaffold: in-vivo and in-vitro Wound Healing Effects
}

\author{
Aaron J. Tabor ${ }^{1,4}$, Andrew Robinson ${ }^{4}$, Bronson I. Pinto ${ }^{1}$ and Robert S. Kellar ${ }^{1,2,3,4 *}$ \\ ${ }^{1}$ Department of Biological Sciences, Flagstaff, AZ 86011, USA \\ ${ }^{2}$ Department of Mechanical Engineering, Flagstaff, AZ 86011, USA \\ ${ }^{3}$ Center for Bioengineering Innovation, Flagstaff, AZ 86011, USA \\ ${ }^{4}$ Development Engineering Sciences, Flagstaff, AZ 86004, USA
}

Received: February 18, 2016; Accepted: March 10, 2016; Published: April 12, 2016

*Corresponding author: Robert S. Kellar, Ph.D, Associate Professor of Practice, Center for Bioengineering Innovation (CBI), Department of Biological Sciences, Department of Mechanical Engineering, Northern Arizona University, 617 South Beaver Street, Bldg. \#21, Box 5640, Flagstaff, AZ 86011, Phone: 928-523-9311; E-mail: robert.kellar@nau.edu

\begin{abstract}
Platelet Rich Plasma (PRP) is an autologous clinical treatment that has been demonstrated to expedite wound healing through the delivery of platelets at a minimum concentration that is three times that of whole blood. PRP is currently used in a variety of clinical applications, including but not limited to: orthodontics, osteogenic care, orthopedics, acute and chronic wounds and various cosmetic applications. Although the treatment has been investigated in many regards, there is a lack of exploration into means of activation through the use of electrospun (e-spun) collagen scaffold technology that allows for activation in situ, or directly in the wound. In this study, this combination therapy (PRP and e-spun collagen scaffolds) was created and investigated first using an in-vitro assay and then in an in-vivo murine full-thickness wound model.

In the current study, a standardized double centrifuge protocol was utilized for the creation of PRP. Flow cytometry and manual counts were performed in order to quantify the degree of platelet concentration. Manual counts yielded a mean platelet count for whole blood and PRP $1 \mu \mathrm{L}$ smears at $10.08 \pm 3.09$ platelets $/ \mu$ land $878.76 \pm 156.28829 .7$ platelets, respectively $(p<0.01)$. Flow cytometry reported a mean platelet count of $426,461 \pm 47,394$ for the processed PRP and a whole blood platelet count of 35,480 $\pm 6,463.75$ ( $<<0.01$ ), supporting that the currently described method produces clinically defined PRP.

Light and scanning electron microscopy were utilized to determine if electrospun collagen scaffolds were an activator of platelets. Analysis was performed using morphological features of the platelets as the activation measurement. Upon application to an electrospun scaffold, platelets presented with an activated state in which the discoid morphology was no longer present, but was replaced with extended pseudopods and blebs. The morphology of non-activated PRP remained discoid and smooth. The change in morphology was indicative of activation and validated that the scaffolds serve as an in-situ platelet activator.
\end{abstract}

An in-vitro scratch assay was utilized as an outcome measure to test the potency and effectiveness of the resulting PRP. The scratch assay has been commonly used as a cellular migration assessment, or in the current study, as a high throughput means to observe in-vitro wound closure. Varying concentrations of PRP were created and applied to a scratched (wounded) fibroblast monolayer in order to determine its effects on the wound. A concentration of $0.25 \%$ PRP was determined to have the highest percent closure at hour 12 compared to the control that did not close until hour 16.

An in-vivo murine full-thickness wound model was used to demonstrate the effectiveness of the PRP and collagen scaffold treatment in a biological model. Post wound creation ( 96 hours) the wound closure percentage of the PRP/electrospun collagen treatment was $100 \%$ compared to the control at $86.076 \pm 11.2806 \%(p=0.01)$, supporting the use of a combination therapy over standardized care. There was no statistical significance between the standalone collagen scaffold treatment and the combination treatment at any time point.

Keywords: Scratch Assay; Wound-Healing,; in-vitro; in-vivo; Platelet-rich plasma; Collagen Scaffolds; Electrospinning

\section{Introduction}

\section{Platelet overview}

Platelets are megakaryocyte cell fragments $2-5 \mu \mathrm{m}$ in diameter with a thickness of $0.5 \mu \mathrm{m}$ and are found circulating in the blood for 8-9 days [1,2]. The platelet has numerous structures and functions that make it critical to the normal healing process in both preventing blood loss and aiding in the cell recruitment and differentiation process.

Platelet formation occurs with the development of a myeloid stem cell into a megakaryocyte through differentiation and a multi-stage maturation. The myloid stem cell differentiates into a megakaryoblast under the influence of thrombopoietin 
(TPO) and undergoes maturation into promegakaryocytes then megakaryocytes, respectively [3, 4]. The megakaryocyte experiences an endo-mitotic process in which cytokinesis fails; this process allows for an increase in ploidy that aids in platelet organelle and granule production [5-7].

While residing in the bone marrow, megakaryocytes are inhibited from forming proplatelets by RhoA activation until they undergo migration to the endothelium where they interact with various extracellular components, e.g. fibrinogen, initiating the proplatelet process [3]. As many as 10-20 proplatelets may form that start as a protrusion, but soon become elongated with branches that fragment into the platelets [8]. The platelet contents, specifically the contents of the alpha granules and dense granules, are of the utmost importance in aiding the progression of wound healing. Once the platelets become activated through collagen exposure in a wound bed, the growth factors and cytokines within the alpha and dense granules are exocytosed. Growth factors include but are not limited to platelet derived growth factor (PDGF), endothelial growth factor (EGF), transforming growth factor-beta (TGF- $\beta$ ) and vascular endothelial growth factor (VEGF) $[9,10]$.

Platelet-rich-plasma (PRP) is an autologous blood product that was originally described as an elevated platelet count above whole blood, and was first used by Dr. Ferrari in 1987 during an open heart surgery [11]. Today the clinical definition of PRP, set by Dr. Robert Marx, is a platelet concentration three times that of peripheral blood [12]. In order to ensure Mark's definition of PRP was being met in the current study, two methods of cellular counting were used; manual counting and cell counts determined by flow cytometry. Manual counting methods are considered an older but useful technique to determine cell concentrations via light microscopy; however, flow cytometry is often considered the standard today. The concentration of platelets as determined by counting methods can be an important factor in determining the quality of PRP with regards to its healing effects due to the relationship to growth factor concentrations.

The premise behind the mechanism of action of PRP is that by increasing the concentration of growth factors and cytokines that are released by platelets, healing will be accelerated; this has been readily demonstrated in the literature $[13,14]$. For clinical purposes, PRP can be applied in either an activated state prior to application or in an inactive state that is physiologically activated within the target location (in situ or on demand activation). Furthermore, it can be utilized as a topical therapeutic agent or delivered as injections to target anatomically deeper tissues.

Electrospun materials have been described since 1934 with patents from Anton Formhals [15-18]. Since then, a number of proteins and polymers have been electrospun into scaffolds for use in both research clinical applications. The process of electrospinning involves the ejection of a solubilized protein through a charged nozzle and onto an oppositely charged target. When the surface tension forces are balanced with the electric field, the protein droplet elongates forming a funnel shape known as a Taylor cone [19]. The stable cone and ejection of the solubilized protein results in the evaporation of the solvent before the target is reached and the formation of nanofibers that are laid upon the target in a nonwoven pattern. These scaffolds have the ability to be used as a wound filling agent and, depending on the protein composition, cellular activators as demonstrated in this study.

The scratch assay has been used as a pre-clinical tool for wound healing since the mid-2000's and provides a method for examining proof of concept [20]. In the current study, human neonatal dermal fibroblasts (hDFn) were utilized, as these cells accurately simulate one of the many cell-cell interactions that take place in the integument involving platelets, growth factors and fibroblasts [21]. Upon reaching confluence, a scratch is performed that represents the creation of a wound. As the hDFn cells begin to fill the scratch, closure of the wound is being represented; thus allowing for a quantifiable in-vitro wound healing assessment to be performed in which various treatments can be applied and analyzed.

In the current study, proof of concept was first tested using an in-vitro assay, which next warranted the use of an in-vivo system in which healing can be observed in a complex living system. In this study a full-thickness murine model was utilized to demonstrate the effectiveness of combining PRP and electrospun (e-spun) collagen scaffolds for the treatment of wounds.

\section{Materials and Methods}

\section{Creation of PRP}

The creation of PRP was based on the methods of Messora and Nagata [22]. A Thermo Scientific ST16R centrifuge, pre-cooled to $4^{\circ} \mathrm{C}$ was used for the current experiments. Porcine whole blood anti-coagulated with acid citrate dextrose (ACD), purchased from Lampire Biological Laboratories (Pipersville, PA.) was distributed aseptically to $50 \mathrm{ml}$ conical tubes in $40 \mathrm{ml}$ aliquots. The whole blood samples were then centrifuged at 200xG for 20 minutes with the acceleration and deceleration of the centrifuge set to 5xG. Upon completion of the initial centrifugation, the buffy coat was transferred to a $15 \mathrm{ml}$ conical tube. The sample underwent a second centrifugation at 400xG for 15 minutes with the acceleration and deceleration of the centrifuge set to $5 \mathrm{xG}$. The finished PRP was extracted and utilized for various analysis.

\section{Creation of Collagen Electrospun Scaffolds}

Lyophilized collagen (Advanced Biomatrix, Carlsbad, CA) was mixed with 1,1,1,3,3,3,-hexafluoro-2-propanol(HFIP) (Oakwood Products, West Columbia,SC)to create a $7.5 \%$ collagen solution. The collagen solution was collected using a syringe and loaded into a syringe pump ( $2 \mathrm{ml}$ of the $7.5 \%$ solution). The 18-gauge blunt end needle of the electrospinner apparatus was set a distance of $12 \mathrm{~cm}$ from an aluminum foil target. The syringe pump was set at a flow rate of $1 \mathrm{ml} / \mathrm{hr}$ and the electrospinner was charged to $25 \mathrm{kV}$ then spun for 1 hour. Following completion, the collagen scaffold was removed from the foil target and cross linked and sterilized using UV light for 1 hour on each side of the scaffold. The sterilization and cross linking used a 15Watt, 254 $\mathrm{nm}$ germicidal UV-Clamp (SpectrolineX-seriesgermicidallamp, Spectronics Corporation, Westbury, NY). 


\section{Manual Counting}

Blood smears were created utilizing $1 \mu$ l of blood with Wright's stain (Carolina Biological Supply Company, Burlington, NC) and washed with $7.0 \mathrm{pH}$ Wrights stain buffer solution (Carolina Biological Supply Company, Burlington, NC). A Leica DM750 with a mounted Leica ICC50 HD camera was used in light microscopic analysis of the smear using methods adapted from Tasker et al. [23]. Ten random fields of view (FOV) in the monolayer were captured at a total magnification of 1000x. The platelets from the ten FOV were counted and averaged for a total of 20 mounted slides: ten whole blood slides and ten PRP slides.

\section{Automated Counting: Flow Cytometry}

PRP $(n=4)$ and whole blood $(n=4)$ platelet concentration were quantified utilizing a BD Accuri C6 flow cytometer. Four varying PRP aliquot volumes were combined with 1x PBS until the volume of the sample reached $1 \mathrm{ml}$, the volumes are outlined below in table 1 . The samples were analyzed at a threshold of 10,000 per recommendations of BD Accuri C6 software users guide (figures 2,3 ).

\section{SEM Analysis}

PRP was diluted at a 1:0.05 1xPBS: PRP ratio to prevent high cell densities that could inhibit analysis. A whole blood and PRP dilution smear was created upon a cover slip using $2 \mu$ of sample. An $8 \mathrm{~mm}$ biopsy punch of a cross linked, electrospun collagen scaffold was used to evaluate the ability for collagen to induce platelet activation. The punch was placed on a cover slip and $2 \mu \mathrm{l}$ of PRP dilution was carefully added to the scaffold in an even distribution causing the scaffold to adhere to the cover slip. All samples were submerged in $2.5 \%$ glutaraldehyde in 1 xPBS overnight at $4^{\circ} \mathrm{C}$. Following overnight fixation, samples were washed three times in 1 xPBS for 5 minutes each. Completion of the washes was followed by submerging the samples in $1 \%$ osmium tetraoxide for 1 hour as a secondary fixative. The samples were then rinsed with DI water three times for 5 minutes each and underwent serial dehydration as follows: $30 \% \mathrm{EtOH}$ for 5 minutes, $50 \%$ EtOH for 5 minutes, $70 \%$ EtOH for 5 minutes, two rounds at $95 \%$ for 10 minutes each, and finally three rounds at $100 \%$ for 10 minutes each. The samples were first immersed in a 1:1 solution of $100 \%$ EtOH: HMDS for 15 minutes followed by a second round with $100 \%$ HMDS for 15 minutes. The solution was then drained and the samples were allowed to air dry overnight. The following morning, the samples were mounted on stubs using carbon tape and were sputter coated with palladium for 10 seconds. Analysis of the samples was performed using a Zeiss Supra 40VP FESEM (Carl-Zeiss-Strasse, Oberkochen, Germany) at a KV of 3.00 and a magnification between $3.67 \mathrm{~K}$ and $7.05 \mathrm{~K}$.

\section{Scratch Assay}

Human neo-natal dermal fibroblasts (hDFn, $3^{\text {rd }}$ passage, (Life Technologies, Carlsbad, CA) were seeded as a monolayer into a T75 culture flask (Corning Inc., Corning, NY.) at a density of 5,000 cells $/ \mathrm{cm}^{2}$. Cells were incubated at $37^{\circ} \mathrm{C}, 5 \% \mathrm{CO}_{2}$ until optimal cell density was achieved ( $>60 \%$ confluence). Cells were then sub-cultured into a tissue culture-treated 12-well plate (Corning,

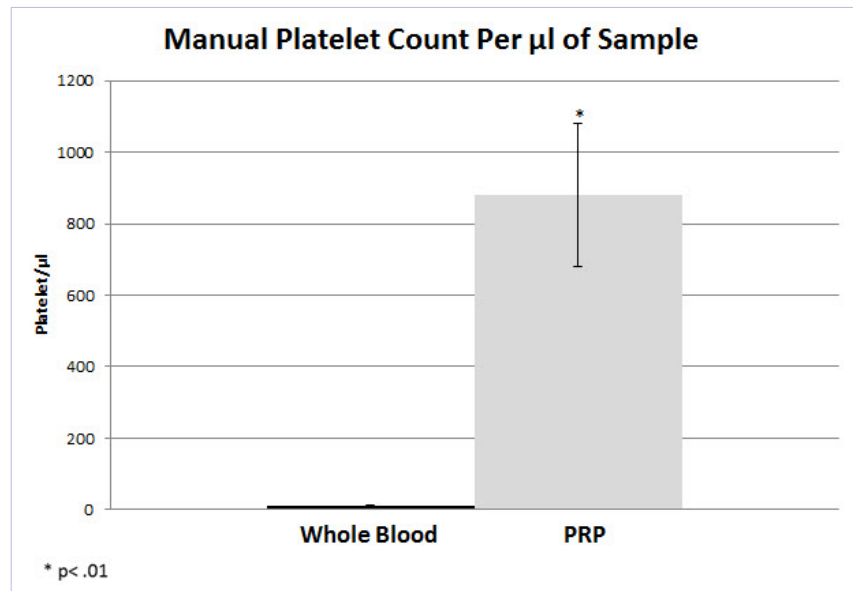

Figure 1: Manual platelet counts between whole blood smears and PRP smears.

Table 1: Dilution factors for flow cytometry.

\begin{tabular}{|c|c|c|}
\hline Run & $\begin{array}{c}\text { Amount whole blood/PRP used } \\
(\boldsymbol{\mu l})\end{array}$ & Volume 1x PBS $(\boldsymbol{\mu l})$ \\
\hline 1 & 1 & 999 \\
\hline 2 & 3 & 997 \\
\hline 3 & 5 & 995 \\
\hline 4 & 7 & 993 \\
\hline
\end{tabular}

Flow Cytometry Platelet Counts

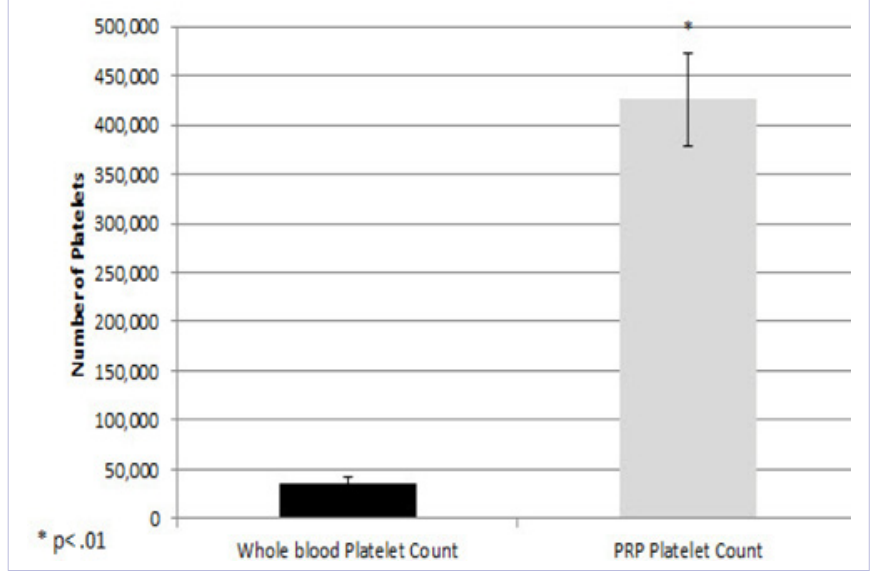

Figure 2: Platelet concentrations of whole blood and PRP based on dilutions.

Inc., Corning NY). Cells were incubated at $37^{\circ} \mathrm{C}, 5 \% \mathrm{CO}_{2}$ for 72 hours or until the cells reached $100 \%$ confluence. Upon reaching confluence, the cells were scratched using a p200 pipet tip (Gilson Inc., Middleton, WI) in a diagonal fashion across the monolayer. The scratch width measured $1.5 \mathrm{~mm} \pm 0.5 \mathrm{~mm}$. Concentrations of PRP at $0.25 \%(n=3), 0.125 \%(n=6), 0.063 \%(n=6), 0.031 \%(n=6)$ and $0.016 \%(n=6)$ were created using a $100 \%$ stock solution of PRP and a stock Dulbecos Modified Eagle Medium (DMEM, Life 
Technologies, Carlsbad, CA.) supplemented with $10 \%$ fetal bovine serum (FBS, Life Technologies, Carlsbad, CA.). The control scratch $(\mathrm{n}=6)$ measured the same width $1.5 \mathrm{~mm} \pm 0.5 \mathrm{~mm}$ and included the same stock Dulbecos Modified Eagle Medium (DMEM, Life Technologies, Carlsbad, CA.) supplemented with $10 \%$ fetal bovine serum (FBS, Life Technologies, Carlsbad, CA.) however it did not contain the PRP. These specific concentrations were used in order to effectively create an environment where PRP would interact with the hDFn cells while also preventing the mixed solution from forming a clot, as a result of the platelet-clotting cascade, which would interfere with qualitative and quantitative outcome measures in this experiment. The various working concentrations were individually and immediately applied to the scratched monolayer of hDFn cells and returned to the incubator for future observation. Images were captured at $0,4,8,12$ and 16 hours respectively using OptixCam image software (OCView V7.3.1.8, The Microscope Store, LLC. Roanoke, VA.) on an Olympus CK2 inverted microscope (Olympus Corporation, Waltham, MA.). Photos were analyzed using ImageJ (V1.48) analysis software (National Institute of Health, Bethesda, MD.)

\section{in-vivo study}

One liter of Porcine ACD-Whole blood was purchased from Lampire Biological Laboratories. The blood arrived one day prior to surgery and contained a pathogen free microbial report. The morning of surgery, PRP was created using the standard creation method as listed above. PRP and whole blood samples were prepared for automated counts to ensure adequate platelet concentration numbers in the whole blood and PRP respectively. Additionally, PRP and whole blood smears were created and viewed using light microscopy, following the wright staining protocol, to ensure appropriate platelet morphology prior to surgical use.

A full-thickness murine hairless-SCID model was used for these studies following Northern Arizona University (NAU) IACUC approval, Protocol 12-006R1. Four, 8 week-old female mice were purchased from Jackson Laboratories model \#002019. The mice arrived in healthy condition at NAU's Research Annex and were allowed a 3 day acclimation period. The surgical study followed a 6-day research plan: day $0=$ full thickness wound creation; day 6 = euthanasization. The pre-operative treatment followed NAU's "Rodent and Small Animal Survival Surgery" SOP No. AC-15-0039. The mice were weighed and anesthetized via inhalation of Isoflurane, to effect. 1-2\% isoflurane in oxygen. This was followed by a pre-operative Buprenorphine $(0.01 \mathrm{mg} /$ $\mathrm{kg}$ ) dose delivered subcutaneously. The full-thickness wounds were created on the dorsum of each animal using a sterile $6 \mathrm{~mm}$ dermal punch and tissue excision following previously published methods in the lab [24]. To mitigate animal harm the dermal punch was utilized to score the integument followed by tissue excision with sterile surgical scissors. The following treatments were randomly applied to the wounds: collagen scaffold, PRP and collagen scaffold, and control (xeroform covered wound) ( $n=5$ for all treatment groups). The animals were then wrapped with a non-adhesive gauze pad and coban wrap to prevent animal interference with wound sites. The bandage dressings were changed every 48 hours at photo time points (days 2 and 4 of the 6 day study) ensuring the wounds were not infected.

Assessment of wounds was performed using photo images at time points 0,48 hours, 96 hours and 144 hours post wound creation. The images were then analyzed with NIH ImageJ software for percent wound closure.

\section{Statistical Analysis}

All statistical analysis were performed using R-Program (V3.2.2 GUI). Student's T-test was used for manual counts and flow cytometry $(p<0.05)$. The scratch assay was evaluated using a one-way ANOVA ( $\mathrm{p}<0.05)$ with a Tukey-Post-HOC test identifying statistical significance between parametric data. invivo studies also used one-way ANOVA with a Tukey-Post-HOC test $(\mathrm{p}<0.05)$.

\section{Results}

\section{Platelet Counts: Manual and Automated}

Mean platelet counts for whole blood and PRP were $10.08 \pm$ 3.09 platelets $/ \mu \mathrm{l}$ and $878.76 \pm 156.28$, respectively. The manual counts demonstrate that the creation method produces PRP according to the Marx definition of greater than $3 \mathrm{x}$ platelet concentration of whole blood.

Manual counting methods were validated through flow cytometry which demonstrated a significant increase ( $p<$ 0.01 ) in platelet concentration in PRP, $426,461 \pm 47,394$, when compared to that of whole blood, $35,480 \pm 6,463.75$. Figures 1 and 2 show the average whole blood and PRP platelet counts using manual and flow cytometry methods. Both counting methods demonstrated that the created PRP contained a platelet concentration above the three fold minimum compare whole blood. Based on Marx's definition, it can be concluded that clinical PRP results from the current methods.

\section{SEM and Light Microscopy Analysis}

The whole blood and PRP samples both showed platelets with a normal morphology, signifying that they were inactivated, as qualitative indications of activation were absent (figure 3 images $A$ and $B$ respectively). This demonstrates that the PRP creation method is not inducing platelet activation through shearing thus allowing for future activation on demand when it will be clinically needed. The platelets that were placed on the collagen scaffold sample exhibited morphological changes associated with platelet activation (figure 3 image C, figure 4, figure 5, light microscopy images). Upon activation, platelets had undergone pseudopod formation and in some cases had visible blebs on their surface; figure 4, supporting that exocytosis, likely of growth factors and cytokines contained in the granules, was occurring. This supports the idea that the PRP can be activated on demand, through the use of electrospun scaffolds, and is thus appropriate for clinical use.

\section{Scratch Assay}

Initial scratch widths measured $1.5 \mathrm{~mm} \pm 0.5 \mathrm{~mm}$. Percent closure was calculated for the scratch assay to determine the 


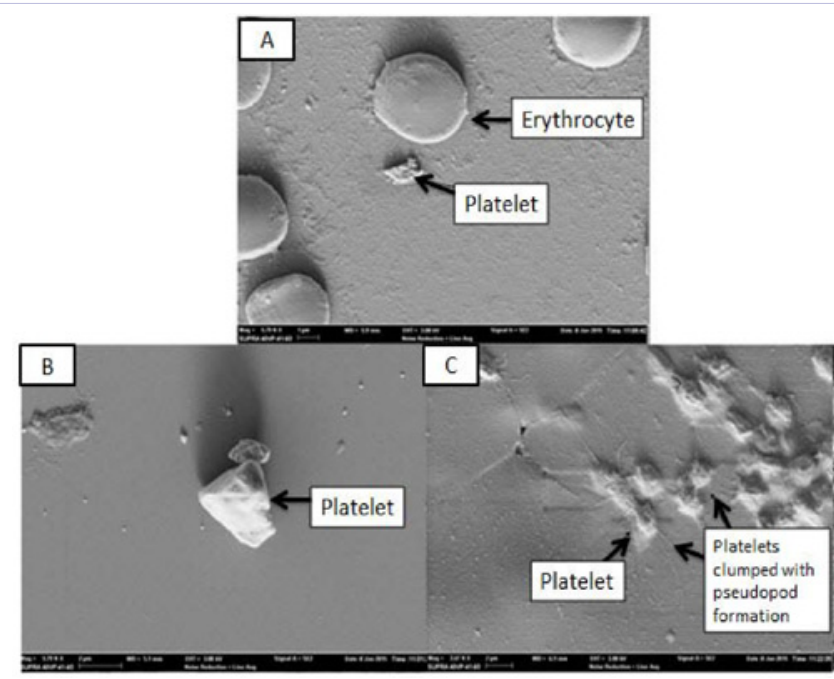

Figure 3: Image A: shows whole blood with a platelet exhibiting normal morphology indicating that it is in an inactivated state. Image B: shows a morphologically normal platelet from the PRP dilution demonstrating that the platelet is inactivated. Image C: shows the PRP and electrospun collagen sample with platelets displaying the classical signs of activation: pseudopod formation.

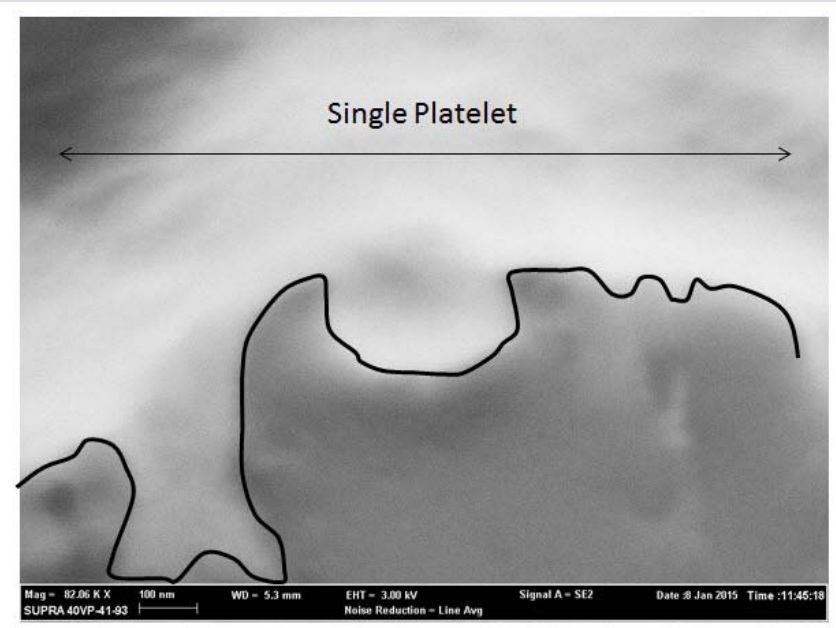

Figure 4: SEM image of activated platelet within the PRP/electrospun collagen sample demonstrating blebbing of vesicle.

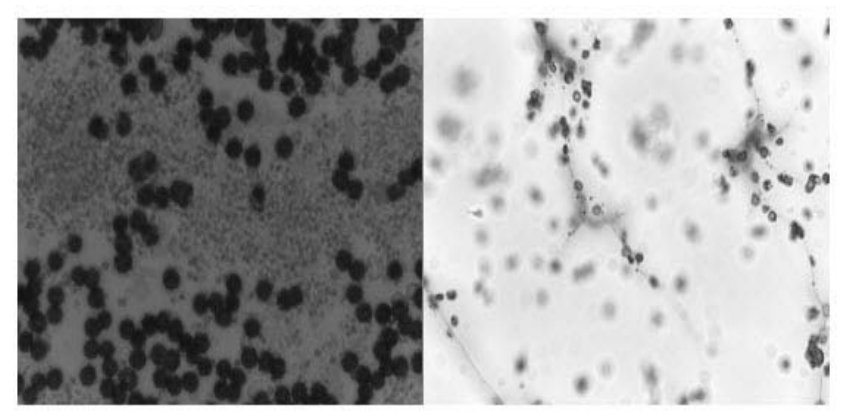

Figure 5: Light microscopy images demonstrating inactive PRP (left) and activated PRP using the collagen scaffold (right). Wright stain in each histology slide (100x mag).

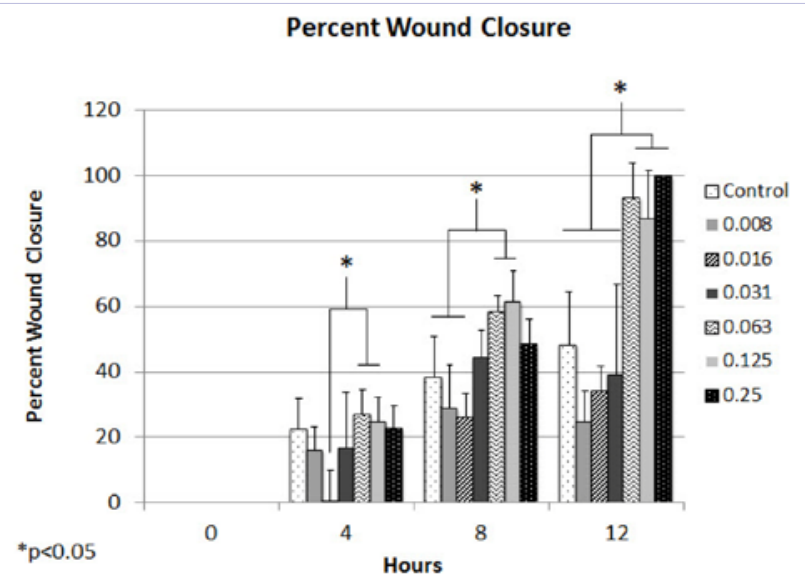

Figure 6: Percent wound closure for in-vitro wound healing model. Statistical significance was determined at timepoints 4,8 and 12 between higher and lower dosages of PRP, including control.

\section{Percent Closure 48 Hours Post Wound Creation}

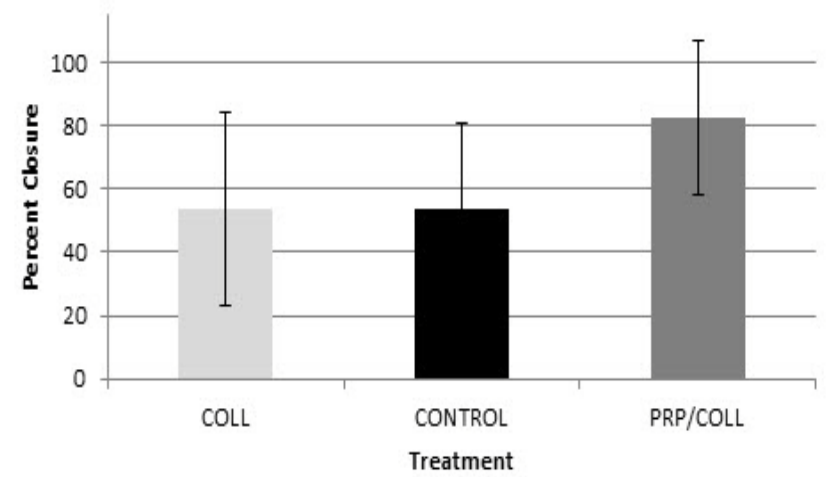

Figure 7: Percent wound closure for in-vivo wound at 48 hours. No statistical significance was present but PRP and electrospun collagen had greater percent closure $82.73 \%$ compared to collagen and control $53.86 \%, 53.40 \%$ respectively.

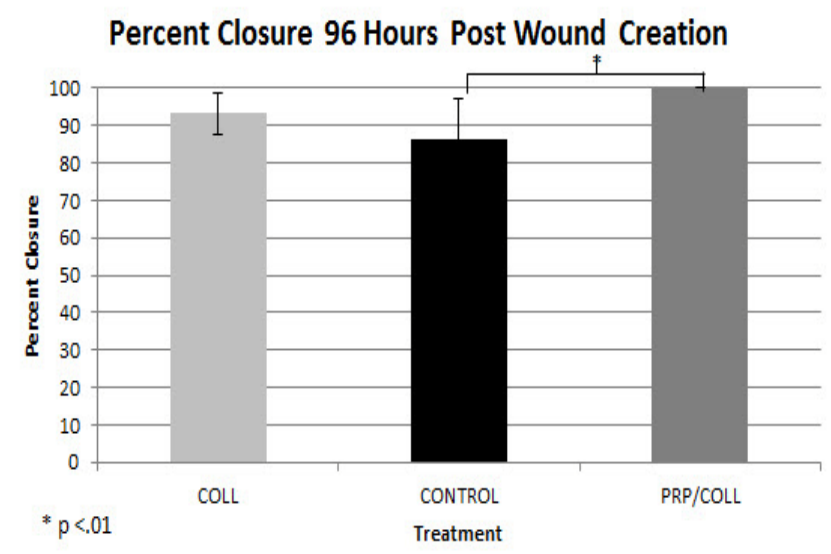

Figure 8: Percent wound closure for in-vivo wound at 96 hours. Statistical significance was present between control and PRP with the electrospun scaffold. 
percent closure at each time point. The $0.25 \%$ PRP treatment had a $100 \%$ closure at the 12 hour compared to control which was approximately $50 \%$ closed at hour 12 . Statistical differences $(\mathrm{p}<$ 05 ) were measured between the higher PRP dosages compared to lower dosages at the standardized time points (figure 6).

\section{in-vivo Study}

The in vivo assessment evaluated two treatment groups and their effects on the progression of wound healing, relative to a control group treated with standardized clinical care. Figures 7 , 8 and 9 detail the findings at the set time points. At 96 hours post wound creation the combination therapy, PRP with electrospun collagen scaffolds, had a percent closure of $100 \%$ compared to the control at $86.076 \pm 11.28 \%(p<0.01)$. At the same time point, the standalone collagen treatment had a mean closure of $93.15 \pm$ $5.66 \%$ and did not demonstrate statistical significance compared to the control. The same trends were seen at 144 hours post wound creation with the combination treatment having a percent closure of $100 \%$ while the control had a percent closure of 96.48 $\pm 2.724 \%(p<0.02)$. The standalone collagen treatment had a percent closure of $98.741 \pm 2.340 \%$ and again lacked statistical significance compared to the control.

\section{Discussion}

Platelet-rich-plasma has been clinically used since the 1980's and has been established to expedite the wound healing response in the integument [25] however, little advancement has occurred with the therapeutic since its inception with regards to wound care. In addition, collagen scaffolds have also been widely used clinical as a means to expedite the wound healing response [26] by providing the integument with an important matrix for cellular adhesion [27]. In the current study, improvements for the concentrated treatment modality were investigated by combining the PRP therapeutic and electrospun collagen scaffolds. The invitro analysis included the creation and validation of PRP based on Marx's 2001 definition and testing of the generated PRP using an in vitro wound healing scratch assay prior to progressing into a full thickness in-vivo murine model that has previously been used by our lab and others $[20,28]$.

Dr. Robert Marx originally defined PRP as having a platelet concentration above whole blood. This definition was later revised to having a minimum platelet concentration three times that of an equal volume of whole blood [12]. Concentrating platelets out of whole blood has been previously described to result in an increase in growth factor concentrations [29]. In the current study, both platelet concentration assessments, manual and automated, demonstrated a greater than three-fold increase in platelet concentration compared to whole blood. Additionally, the specific methods described in the current study generated PRP that was not in an activated state. Creating PRP in an inactive state and then activating on demand allows the platelets to provide a wound bed with the highest concentration of growth factors possible with a minimum amount of growth factor degradation or platelet morphology changes [30, 31]. Publications have reported the mode in which PRP is created can have an effect on the amount of cytokine presence [32]. In some cases an improper creation method can allow for growth factor degradation prior to treatment application thus resulting in minimal benefit to wound healing. This could come by way of platelet shearing in which the platelets are shearedopen during the creation process [33] leading to premature activation of the PRP, which could lead to bioactive protein degradation from the platelet granules prior to their desired application.

Based on the published variations in PRP creation modalities and the importance of preserving growth factor activity for the wound, we propose that an updated definition for PRP iswarranted: PRP requires a minimum platelet concentration three times that of whole blood with a creation process that does not cause platelet sheer activation. Sheer activation is the unintended activation/degranulation of platelets due to excessive stress during the centrifugation process. Thisupdated definition of PRP is important because using it could limit the amount of bioactive protein degradation from the creation process leading to maximum concentration of these proteins at the wound bed. To ensure this definition was followed in the current study, two methods were used to determine that the creation method was not causing sheerplatelet activation: an imaging assessment through scanning electron microscopy (SEM) and light microscopy. SEM analysis detailed that platelets of the whole blood and post creation samples maintained normal morphological features and therefore were in an inactivated state. The activated samples, activated with the electrospun collagen scaffold, had a change in morphological characteristic with the formation of pseudopods and blebs indicative of activation. This supports the use of electrospun collagen scaffolds as a platelet activator that can subsequently be utilized as an "on demand" activator of PRP. This in-situ collagen activation of platelets occurs through the binding of collagen through glycoprotein VI that causes cytoskeleton changes and secretion of platelet granule contents [34].

Cellular migration assays have been long used to determine how rapidly cells may cover a given area [20,35]. In the current study, the scratch assay, was utilized to determine the percent wound closure in an in-vitro setting. The scratch assay provides a

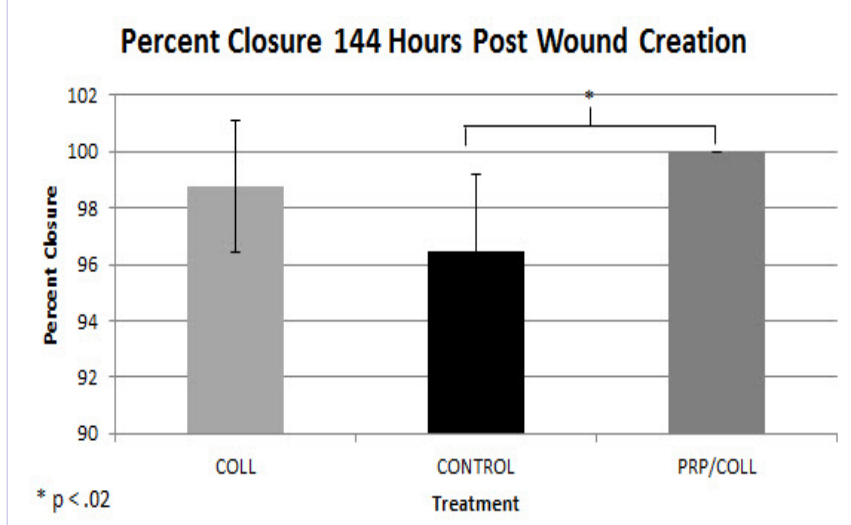

Figure 9: Percent wound closure for in-vivo wound at 144 hours. Statistical significance was present between control and PRP with the electrospun scaffold. Note all PRP with electrospun collagen treatments were $100 \%$ closed compared to control and standalone collagen. 
bench top tool that allows for assessments of various treatments on cell migration (wound closure) that have been described to be superior to many other previously described methods [20]. An ideal cell for this model, the human fibroblast, is utilized due to its importance in the healing progression [36]. Fibroblasts are typically found in the integument and play a significant role in the matrix remodeling and scar formation stages of the wound healing process. Fibroblasts are collagen-secreting cells that work simultaneously with matrix metalloproteinases and other cells to degrade and lay down new extracellular matrix for newly synthesized cells to adhere, proliferate and take residence. Due to these important aspects, fibroblasts offer an accurate in-vitro simulation of in-vivo wound progression when utilizing a single cell type. Varying concentrations of PRP were utilized in the scratch assay in order to determine effects of concentration and also to maintain a visible field of view within the culture. In summary, an increase in concentration led to an increase in percent wound closure of the mock wound compared to the control, likely as a result of the increase in growth factors present with increasing platelet concentration. The highest concentration of PRP, $0.25 \%$, led to largest percent closure at the 12 hour compared to control that closed at 16 hours. In our proposed new PRP definition, the PRP must first be prepared in an inactivated state; therefore, in these experiments that platelets were to be activated in the scratch assay. This was accomplished through the presence of calcium chloride $\left(\mathrm{CaCl}_{2}\right)$ in the culture media. Calcium does not directly activate platelets, but rather aids in the activation of prothrombin to thrombin that is a potent platelet activator, and thus secondary activation of PRP is not needed [37]. In this way, our benchtop scratch assay model afforded the ability to evaluate our "on demand" PRP activation prior to moving into a more complicated full thickness wound model.

Due to the complexity of wound healing in a living system, an in vitro wound healing assessment can only provide limited information, specifically in the current studies we only evaluated how dermal fibroblasts would respond to "on demand" activated PRP. There are numerous additional cell types to fibroblasts that are involved in a complete wound healing response and their complex interactions are difficult to model in an in-vitro model Therefore, in-vivo studies were performed using a murine model to determine the effects the treatments had on the complex healing progression of a full thickness wound. The treatments were as follows: a combination therapy of an electrospun collagen scaffold and PRP, a standalone electrospun collagen scaffold, and a control wound treated with standardized care. The combination therapy (PRP + electrospun collagen) demonstrated statistically significant wound closure percentages compared to control wounds at 96 and 144 hours post wound creation. The standalone treatment (electrospun collagen scaffold) was not statistically different than control wounds at any of the timepoints evaluated. The benefit seen in the combination treatment compared to the control can be attributed to utilizing both a collagen treatment and PRP together in a wound. Thus, multiple agents are being provided to the wound bed that aid in acceleration through the normal wound healing process. The collagen treatment provides the wound bed with a vital skin protein in the form of a matrix in which cells can proliferate and infiltrate. In addition to providing the cells with a matrix that was largely lost in the wound creation, the electrospun collagen scaffold was determined to active the platelets within the PRP treatment. By activating the PRP "on demand" or in location, the growth factors and cytokines immediately become bioavailable to the wound bed within a collagen framework, thus providing the wound with both a matrix architecture and readily bioactive growth factors and cytokines.

Recently published data indicates that the United States spends over $\$ 25$ billion dollars to treat approximately 6.5 million patient wounds [38]. Many of these woundsrequire long clinical interventions and standing care which in turn cause systemic and emotional concerns for patients. The further development and enhancement of wound products such as inactive PRP and activated electrospun collagen scaffold therapies could help to reduce these long term complications, thus improving patient care while making a large economic impact.

\section{Acknowledgements}

1. Aubrey Funke, Assistant Director of Imaging and Histology Core Facility, Northern Arizona University

2. National Science Foundation Grant (NSF \#1126742)

Title: "MRI: Acquisition of a Scanning Electron Microscope for Multi-Disciplinary Research and Training at Northern Arizona University".

3. Robert Diller, Graduate Student, Northern Arizona University

\section{References}

1. Michelson, A. D. Platelets. Amsterdam: Academic Press. 3 rd ed. Netherlands;2013.

2. Harker L, Roskos L, Marzec U, Carter R, Cherry J, Sundell B, et al. Effects of megakaryocyte growth and development factor on platelet production, platelet life span, and platelet function in healthy human volunteers. Blood. 2000;95(8):2514-2522.

3. Bluteau D, Lordier L, Di Stefano A, Chang Y, Raslova H, Debili N, et al. Regulation of megakaryocyte maturation and platelet formation. J thromb Haemost. 2009;7 Suppl 1:227-234. doi: 10.1111/j.15387836.2009.03398.x

4. George, J. Platelets. The Lancet. 2000;355(9214):1531-1539.

5. Lordier L, Jalil A, Aurade F, Larbret F, Larghero J, Debili N, et al. Megakaryocyte endomitosis is a failure of late cytokinesis related to defects in contractile ring and Rho/Rock signaling. Blood. 2008;112(2):3164-3174. doi: 10.1182/blood-2008-03-144956.

6. Geddis A, Kaushansky K. Endomitotic megakaryocytes form a midzone in anaphase but have a deficiency in cleavage furrow formation. Cell Cycle. 2006;5(5):538-545.

7. Raslova H, Roy L, Vourc'h C, Le Couedic J, Brison O, Meltivier D, et al. Megakaryocyte polyploidization is associated with functional gene amplification. Blood. 2003;101(2):541-544.

8. Machlus K, Italiano J. The incredible journey: from megakaryocyte development to platelet formation. 2013;201(6):785-796. doi: $10.1083 /$ jcb.201304054. 
9. Marx RE, Carlson ER, Eichstaedt RM, Schimmele SR, Strauss JE, Georgeff KR. Platelet-rich plasma: growth factor enhancement for bone grafts. Oral Surgery Oral Medicine Oral Pathology Oral Radiology and Endodontology. 1998;85(6):638-646.

10. Maharaj A, \&D'Amore P. Roles for VEGF in the adult. Microvascular Research. 2007:74(2-3):100-113.

11. Ferrari M, Zia S, Valbonesi M, Henriquet F, Venere G, Spagnolo S, et al A new technique for hemodilution, preparation of autologous plateletrich plasma and intraoperative blood salvage in cardiac surgery. Int J Artif Organs. 1987;10(1):47-50.

12. Marx RE. Platelet-rich plasma (PRP):What is PRP and what is not PRP? Implant Dent. 2001;10(4):225-228.

13. Murray MM, Spindler KP, Abreu E, Muller JA, Nedder A, Kelly M, et al Collagen-platelet rich plasma hydrogel enhances primary repair of the porcine anterior cruciate ligament. Journal of Orthopaedic Research 2007;25(1):81-91.

14. Sanchez M, Anitua E, Orive G, Mujika I, Andia I. Platelet-rich therapies in the treatment of orthopaedic sport injuries. Sports medicine. 2009;39(5):345-354. doi: 10.2165/00007256-200939050-00002.

15. Formhals A. Method of producing artificial fibers. 1939;U.S. Patent No. 2158415 .

16. Formals A. Method and apparatus for spinning. 1944;U.S. Patent No. 2349950

17. Formhals A. Process and apparatus for preparing artificial threads. 1934;U.S. Patent No. 1975504.

18. Garg K, Bowlin G. Electrospinning jets and nanofibrous structures. Biomicrofluidic. 2011;5(1):13403. doi: 10.1063/1.3567097.

19. Taylor G. Electrically driven jets. Proc Roy Soc Lond 1969;313(1515)453-475. DOI: 10.1098/rspa.1969.0205.

20. Chun-Chi L, Park A, \& Guan J. In vitro scratch assay: A convenient and inexpensive method for analysis of cell migration in vitro. Nature Protocols. 2007;2(2):329-333.

21. Rifkin DB, Moscatelli D. Recent developments in the cell biology of basic fibroblast growth factor. The Journal of Cell Biology. 1989;109(1):1-6

22. Messora MR, Nagata MJH, Furlaneto FAC, Dornelles RCM, Bomfim SRM., Deliberador TM, et al. A standardized research protocol for platelet-rich plasma (PRP) preparation in rats. RSBO (Online). 2011;8(3):299-304.

23. Tasker S, Cripps P, Mackin A. Evaluation of methods of platelet counting in the cat. Journal of Small Animal Practice. 2001;42(47):326-332.

24. Machula H, Ensley B, Kellar R. Electrospun tropoelastin for delivery of therapeutic adipose-derived stem cells to full-thickness dermal wounds. Advances in wound care. 2014;3(5):367-375.

25. DeRossi R, Coelho AC, Mello GS, Frazílio FO, Leal CR, Facco G G, et al. Effects of platelet-rich plasma gel on skin healing in surgical wound in horses. Acta cirúrgica brasileira. 2009;24(4):276-281.

26. Ruszczak Z. Effect of collagen matrices on dermal wound healing
Advanced drug delivery reviews. 2003;55(12):1595-1611.

27.Zhong S, Teo W E, Zhu X, Beuerman R W, Ramakrishna S,Yung LY. An aligned nanofibrous collagen scaffold by electrospinning and its effects on in vitro fibroblast culture. Journal of Biomedical Materials Research Part A. 2006;79(3):456-463.

28. Walter MN, Wright KT, Fuller HR, MacNeil S, Johnson W E. Mesenchymal stem cell-conditioned medium accelerates skin wound healing: an in vitro study of fibroblast and keratinocyte scratch assays. Experimental cell research. 2010;316(7):1271-1281. doi: 10.1016/j. yexcr.2010.02.026.

29. Eppley B L, Woodell J E, Higgins J. Platelet quantification and growth factor analysis from platelet-rich plasma: implications for wound healing. Plastic and reconstructive surgery. 2004;114(6):1502-1508.

30. Fijnheer R, Pietersz RN, Korte DD, Gouwerok CW, Dekker WJ, Reesink HW, et al. Platelet activation during preparation of platelet concentrates: a comparison of the platelet-rich plasma and the buffy coat methods. Transfusion. 1990;30(7):634-638.

31. Metcalfe P, Williamson LM, Reutelingsperger CP, Swann I, Ouwehand WH, Goodall A H. Activation during preparation of therapeutic platelets affects deterioration during storage: a comparative flow cytometric study of different production methods. British journal of haematology. 1997;98(1):86-95.

32. Wadhwa M, Seghatchian MJ, Lubenko A, Contreras M, Dilger P, Bird C, et al. Cytokine levels in platelet concentrates: quantitation by bioassays and immunoassays. British journal of haematology. 1996;93(1):225-234.

33. Bausset O, Giraudo L, Veran J, Magalon J, Coudreuse JM, Magalon G, et al. Formulation and storage of platelet-rich plasma homemade product. BioResearch open access. 2012;1(3):115-123. doi: 10.1089/ biores.2012.0225.

34. Kehrel B, Wierwille S, Clemetson KJ, Anders O, Steiner M, Knight CG, et al. Glycoprotein VI is a major collagen receptor for platelet activation: it recognizes the platelet-activating quaternary structure of collagen, whereas CD36, glycoprotein IIb/IIIa, and von Willebrand factor do not. Blood. 1998;91(2):491-499.

35. Yarrow JC, Perlman ZE, Westwood NJ, Mitchison TJ. A high-throughput cell migration assay using scratch wound healing, a comparison of image-based readout methods. BMC biotechnology. 2004;4:21. DOI: $10.1186 / 1472-6750-4-21$.

36. Martin P. Wound healing--aiming for perfect skin regeneration. Science. 1997;276(5309):75-81.

37.Yip C, Linden M, Attard C, Monagle P, Ignjatovic V. Platelets from children are hyper-responsive to activation by thrombin receptor activator peptide and adenosine diphosphate compared to platelets from adults. British Journal of Hematology. 2014;168(4):526, 532. doi: $10.1111 /$ bjh.13153.

38. Sen CK, Gordillo GM, Roy S, Kirsner R, Lambert L, Hunt TK, et al. Human Skin Wounds: A Major and Snowballing Threat to Public Health and the Economy. Wound Repair and Regeneration. 2009;17(6):763-771. doi:10.1111/j.1524-475X.2009.00543.x 